\title{
TRANSLATION INVARIANT IDEALS
}

\author{
SŁAWOMIR SOLECKI
}

\begin{abstract}
Translation invariant ideals of subsets of groups and their invariant extensions are studied.
\end{abstract}

\section{INTRODUCTION}

We investigate invariant extensions of left translation invariant ideals on groups and prove a general theorem on such extensions. As a consequence of it, we show that the Haar measure on any locally compact, second countable, Abelian group $G$ admits a translation invariant extension which measures at least one selector of the family of cosets of any uncountable subgroup of $G$. This extends a result of Nowik [11] and complements some earlier results of the author [14]. We show how a modification of a technique of Erdős, Kunen, Mauldin, Friedman, and Talagrand [4, 6] answers a question of Cichon by proving that under Martin's axiom there exists a subgroup of $\mathbb{R}$, the reals, of cardinality continuum whose all selectors are not Lebesgue measurable and do not have the property of Baire. Additionally, again as a consequence of our general result, we prove that for a regular cardinal $\kappa$, any Abelian group $G$ carries a translation invariant ideal $\mathcal{J}$ with the property that for $X \subseteq G,|X|<\kappa$ if, and only if, $X$ can be translated into the complement of any set from $\mathcal{J}$. This answers a question of Seredyński [12].

I would like to clarify basic notions now. Let $G$ be a group, let $H$ be a subgroup of $G$. By right cosets of $H$ we mean cosets of the form $H g$ for $g \in G$. If $A$ is a subset of $G$ which is the union of a family of right cosets of $H, A / H$ denotes the family of all the right cosets contained in $A$. We say that $S \subseteq G$ is a selector of $A / H$ if $S$ is a subset of $A$ and picks precisely one point from each right coset of $H$ included in $A$. If $A$ happens to be empty, the only selector of $A / H$ is $\emptyset$. Sometimes selectors of $G / H$ will be called selectors of $H$. While studying left translation invariant measures and ideals on $G$, we consider right cosets of $H$ since these are orbits of the action of $H$ on $G$ by left translations. Therefore, problems considered here can be reformulated for the more general setting where $G$ acts on a set $X$ and the invariant measure is defined on $X$ rather than on $G$. This setting is adapted, for example, in [14].

For subsets $A, B$ of a group $G, A B$ denotes $\left\{g_{1} g_{2}: g_{1} \in A, g_{2} \in B\right\}$. $\mathbb{N}$ stands for the set of all natural numbers including 0 .

1991 Mathematics Subject Classification. 03E05, 28C10, 28E15, 43A10.

Key words and phrases. Translation invariant measures, translation invariant ideals, selectors, extensions of measures.

Research supported by NSF grant DMS-9803676 


\section{A GENERAL THEOREM}

Theorem 1.1. Let $\mathcal{I}$ be a family of subsets of a group $G$ such that $|\mathcal{I}| \leq|G|$ and $|G \backslash X|=|G|$ for any $X \in \mathcal{I}$. Let $\kappa \leq|G|$ be a regular cardinal. Assume that for any subgroup $H$ of $G$ and $a \in G,|\langle H \cup\{a\}\rangle / H|<\kappa$.

Then there exists a family $\mathcal{F}$ of subsets of $G$ such that

(i) if $\mathcal{A} \subseteq \mathcal{F}$ and $X \subseteq G$ are such that $|\mathcal{A}|,|X|<\min (\kappa, \operatorname{cof}(|G|))$, then $G \backslash$ $X(\bigcup \mathcal{A})$ is not contained in a member of $\mathcal{I}$;

(ii) if $X \subseteq G$ and $|X| \geq \kappa$, then there exists $A \in \mathcal{F}$ with $X A=G$;

(iii) if $X \subseteq G$ and $|X|<\kappa$, then for any $A_{0}, A_{1}, \ldots, A_{n} \in \mathcal{F}, n \in \mathbb{N}$, we have $\left(\bigcup_{i \leq n} A_{i}\right) X \neq G$.

Proof. For an ordinal $\delta$, a sequence $\mathcal{H}=\left\langle H_{\gamma}: \gamma<\delta\right\rangle$ of subgroups of $G$ is called a $\delta$-tower of groups if $\bigcup_{\xi<\gamma} H_{\xi} \subseteq H_{\gamma}$ and $\bigcup_{\xi<\gamma} H_{\xi} \neq H_{\gamma}$. We will always have, and we make it a part of the definition of $\delta$-tower of groups, that $\operatorname{cof}(\delta)=\kappa$. $\delta$ is called the height of the tower. If we do not want to specify the height of a $\delta$-tower, we will call it simply a tower. We write $H$ for the group $\cup \mathcal{H}$. We also adapt the convention that if a $\delta$-tower of groups is denoted by $\mathcal{H}^{\alpha}$, then its elements are denoted by $H_{\gamma}^{\alpha}, \gamma<\delta$, and $\cup \mathcal{H}^{\alpha}$ by $H^{\alpha}$. If $\mathcal{H}^{1}$ is a $\delta_{1}$-tower of groups and $\mathcal{H}^{2}$ are $\delta_{2}$-tower of groups, put

$$
\mathcal{H}^{1}<\mathcal{H}^{2} \Leftrightarrow H^{1} \subseteq H_{\gamma}^{2} \text { for some } \gamma<\delta_{2}
$$

Given a $\delta$-tower $\mathcal{H}$, let $S \subseteq G$ be a selector for $G / H$, that is, $H S=G$ and, for any two different $g_{1}, g_{2} \in S, g_{2} g_{1}^{-1} \notin H$. For a subset $A \subseteq \delta$ let

$$
S(A)=\bigcup_{\alpha \in A}\left(H_{\alpha} \backslash \bigcup_{\gamma<\alpha} H_{\gamma}\right) S .
$$

Let $\mathcal{N}$ be an ideal of subsets of $\delta$. Define

$$
\mathcal{J}(\mathcal{H}, S, \mathcal{N})=\{S(A): A \in \mathcal{N}\}
$$

An ideal of subsets of $\delta$ will be called acceptable if it fulfills the following three properties: the union of $<\kappa$ sets from $\mathcal{N}$ is in $\mathcal{N}$ (i.e., $\mathcal{N}$ is $\kappa$-complete); $\delta \backslash A$ is cofinal in $\delta$ for any $A \in \mathcal{N}$; for any $B \subseteq \delta$ cofinal in $\delta$ there is $A \subseteq B$ with $A \in \mathcal{N}$ and $A$ cofinal in $\delta$. We will construct an increasing, with respect to $<$, sequence $\mathcal{H}^{\alpha}, \alpha<\rho$ for some ordinal $\rho$, of towers of groups, selectors $S_{\alpha}$ for $G / H^{\alpha}$, and acceptable ideals $\mathcal{N}_{\alpha}$, and then define

$$
\mathcal{F}=\bigcup_{\alpha<\rho} \mathcal{J}\left(\mathcal{H}^{\alpha}, S_{\alpha}, \mathcal{N}_{\alpha}\right)
$$

First, we show that $\mathcal{F}$ defined in this fashion for an arbitrary increasing sequence of towers of groups fulfills (iii) from Theorem 1.1. Later, we will specify what additional conditions the $\mathcal{H}^{\alpha}$ 's must fulfill in order that (ii) be satisfied as well. Finally, we will construct a sequence $\mathcal{H}^{\alpha}$ along with $S_{\alpha}$, and $\mathcal{N}_{\alpha}, \alpha<\rho$, which satisfy these conditions and are such that $\mathcal{F}$ additionally fulfills (i). 
Claim 1. Let $\mathcal{H}^{\alpha}, \alpha<\rho$, be an increasing sequence of towers of groups with $\mathcal{H}^{\alpha}$ of height $\delta_{\alpha}$, let $S_{\alpha}$ be a selector for $G / H^{\alpha}$ and let $\mathcal{N}_{\alpha}$ be an acceptable ideal of subsets of $\delta_{\alpha}$. Then $\mathcal{F}$ fulfills (iii).

Proof. It is enough to show that the conclusion holds when the sequences are finite, that is, we have $\mathcal{H}^{1}<\cdots<\mathcal{H}^{n}$ of height $\delta_{1}, \ldots, \delta_{n}$, respectively, $S_{1}, \ldots, S_{n}$, and $\mathcal{N}_{1}, \ldots, \mathcal{N}_{n}$ for some $n \in \mathbb{N}$.

First note that if $|Y|<\kappa$ and $S(A) \in \mathcal{J}(\mathcal{H}, S, \mathcal{N})$ with $\mathcal{H}$ of height $\delta$, then there exist unboundedly many $\alpha<\delta$ such that $h Y \cap S(A)=\emptyset$ for any $h \in H_{\alpha} \backslash \bigcup_{\gamma<\alpha} H_{\gamma}$. Indeed, since $\operatorname{cof}(\delta)=\kappa$, we can find $\gamma_{0}<\delta$ with $Y \subseteq H_{\gamma_{0}} S$. Any $\alpha>\gamma_{0}, \alpha \notin A$ works, since if $h \in H_{\alpha} \backslash \bigcup_{\gamma<\alpha} H_{\gamma}$, then

$$
h Y \subseteq h H_{\gamma_{0}} S \subseteq\left(H_{\alpha} \backslash \bigcup_{\gamma<\alpha} H_{\gamma}\right) S \subseteq S(\delta \backslash A),
$$

and clearly $S(\delta \backslash A) \cap S(A)=\emptyset$.

Now let $X \subseteq G$ have cardinality less than $\kappa$, and let $A_{1} \in \mathcal{N}_{1}, \ldots, A_{n} \in \mathcal{N}_{n}$. To establish (iii), we need to show that, for some $h \in G, h X^{-1} \cap \bigcup_{i=1}^{n} S_{i}\left(A_{i}\right)=\emptyset$. Pick $\alpha_{n}<\delta_{n}$ with $\bigcup_{i=1}^{n-1} H^{i} \subseteq H_{\alpha_{n}}^{n}$. Pick $\alpha_{n}^{\prime}>\alpha_{n}$ from the unbounded set as above (for $S_{n}\left(A_{n}\right)$ and $Y=X^{-1}$ ). Let $h_{n} \in H_{\alpha_{n}^{\prime}}^{n} \backslash \bigcup_{\gamma<\alpha_{n}^{\prime}} H_{\gamma}^{n}$. Note that for any $h \in \bigcup_{i=1}^{n-1} H^{i}$, we have $h h_{n} \in H_{\alpha_{n}^{\prime}}^{n} \backslash \bigcup_{\gamma<\alpha_{n}^{\prime}} H_{\gamma}^{n}$, whence

$$
h h_{n} X^{-1} \cap S_{n}\left(A_{n}\right)=\emptyset \text {. }
$$

Next find $\alpha_{n-1}<\delta_{n-1}$ such that $\bigcup_{i=1}^{n-2} H^{i} \subseteq H_{\alpha_{n-1}}^{n-1}$. Pick $\alpha_{n-1}^{\prime}>\alpha_{n-1}$ from the unbounded set constructed above (for $S_{n-1}\left(A_{n-1}\right)$ and $Y=h_{n} X^{-1}$ ). Let $h_{n-1} \in H_{\alpha_{n-1}^{\prime}}^{n-1} \backslash \bigcup_{\gamma<\alpha_{n-1}^{\prime}} H_{\gamma}^{n-1}$. Then for any $h \in \bigcup_{i=1}^{n-2} H^{i}$, we have

$$
h h_{n-1} h_{n} X^{-1} \cap\left(S\left(A_{n-1}\right) \cup S\left(A_{n}\right)\right)=\emptyset .
$$

In the same fashion we construct $h_{n-2}, \ldots, h_{1}$ and, finally, put $h=h_{1} h_{2} \cdots h_{n}$. This finishes the proof of the claim.

Now we specify properties of an increasing sequence of towers of groups needed to prove (ii). Let $\mathcal{H}^{\alpha}, \alpha<\rho$, be an increasing sequence of towers of groups with $\mathcal{H}^{\alpha}$ of height $\delta_{\alpha}$.

(a) $\bigcup_{\alpha<\rho} H^{\alpha}=G$;

(b) if $\operatorname{cof}(\alpha) \neq \kappa$, then $H_{0}^{\alpha} \subseteq \bigcup_{\gamma<\alpha} H^{\gamma}$ for $\alpha>0$ and $H_{0}^{0}=\{1\}$ and, for any $\gamma<\delta_{\alpha},\left|H_{\gamma}^{\alpha} / H_{0}^{\alpha}\right|<\kappa$

(c) if $\operatorname{cof}(\alpha)=\kappa$, then $\forall \gamma<\kappa \exists \beta<\alpha H_{\gamma}^{\alpha} \subseteq H^{\beta}$;

(d) $\operatorname{cof}(\rho) \neq \kappa$.

Claim 2. Let $\mathcal{H}^{\alpha}, \alpha<\rho$, be a sequence of towers of groups with properties (a)-(d). Let $S_{\alpha}$ be a selector for $G / H^{\alpha}$ and let $\mathcal{N}_{\alpha}$ be an acceptable ideal of subsets of $\delta_{\alpha}$. Then $\mathcal{F}$ fulfills (ii).

Proof. If $\mathcal{H}$ be a $\delta$-tower of groups, we say that a set $Y$ is unbounded in $\mathcal{H}$ if there does not exist $\gamma<\delta$ such that $Y \cap H \subseteq H_{\gamma}$. First we will show that if $Y$ is unbounded in a $\delta$-tower of groups $\mathcal{H}$ with a selector $S$ for $G / H$ and an acceptable ideal $\mathcal{N}$ on $\delta$, then, for some $A \in \mathcal{N}, Y g \cap S(A) \neq \emptyset$ for any $g \in G$. Let 
$B=\left\{\alpha \in \delta: Y \cap\left(H_{\alpha} \backslash \bigcup_{\gamma<\alpha} H_{\gamma}\right) \neq \emptyset\right\}$. Then $B$ is cofinal in $\delta$, and we can find a cofinal set $A \in \mathcal{N}$ with $A \subseteq B$. Let $g \in G$. Find $s \in S$ such that $g s^{-1} \in H$. Pick $\alpha \in A$ with $g s^{-1} \in H_{\gamma}$ for some $\gamma<\alpha$. Then since $Y \cap\left(H_{\alpha} \backslash \bigcup_{\gamma<\alpha} H_{\gamma}\right) \neq \emptyset$,

$$
\emptyset \neq Y g s^{-1} \cap\left(H_{\alpha} \backslash \bigcup_{\gamma<\alpha} H_{\gamma}\right) g s^{-1}=Y g s^{-1} \cap\left(H_{\alpha} \backslash \bigcup_{\gamma<\alpha} H_{\gamma}\right)
$$

Hence

$$
\emptyset \neq Y g \cap\left(H_{\alpha} \backslash \bigcup_{\gamma<\alpha} H_{\gamma}\right) s \subseteq Y g \cap S(A) .
$$

Now let $X \subseteq G$ have cardinality greater than or equal to $\kappa$. We need to find $\alpha_{0}<\rho$ and $A \in \mathcal{N}_{\alpha_{0}}$ such that, for any $g \in G, X^{-1} g \cap S_{\alpha_{0}}(A) \neq \emptyset$. By what was proved above, it is enough to find $\alpha_{0}<\rho$ and $g_{0} \in G$ such that $X^{-1} g_{0}$ is unbounded in $\mathcal{H}^{\alpha_{0}}$. Let

$$
\alpha_{0}=\min \left\{\alpha<\rho: \exists g \in G\left|X^{-1} g \cap H^{\alpha}\right| \geq \kappa\right\} .
$$

The ordinal $\alpha_{0}$ is well-defined by (a) and (d) since the sequence $\mathcal{H}^{\alpha}, \alpha<\rho$, is increasing. Pick $g_{0} \in G$ with $\left|X^{-1} g_{0} \cap H^{\alpha_{0}}\right| \geq \kappa$.

If $\operatorname{cof}\left(\alpha_{0}\right) \neq \kappa$, then for any $h \in G,\left|X^{-1} g_{0} h^{-1} \cap \bigcup_{\gamma<\alpha_{0}} H^{\gamma}\right|<\kappa$ since otherwise we would have $\left|X^{-1} g_{0} h^{-1} \cap H^{\alpha}\right| \geq \kappa$ for some $h \in G$ and some $\alpha<\alpha_{0}$ contradicting the choice of $\alpha_{0}$. Hence, by (b),

$$
\left|X^{-1} g_{0} \cap H_{0}^{\alpha_{0}} h\right|<\kappa \text { for any } h \in G .
$$

By (b) again, each $H_{\gamma}^{\alpha_{0}}, \gamma<\delta_{\alpha}$, is the union of less than $\kappa$ many right cosets of $H_{0}^{\alpha_{0}}$. Thus, by (1), we get that $\left|X^{-1} g_{0} \cap H_{\gamma}^{\alpha_{0}}\right|<\kappa$ for any $\gamma<\delta_{\alpha_{0}}$, whence $X^{-1} g_{0}$ is unbounded in $\mathcal{H}^{\alpha_{0}}$.

If $\operatorname{cof}\left(\alpha_{0}\right)=\kappa$, then, since $\left|X^{-1} g_{0} \cap H^{\beta}\right|<\kappa$ for any $\beta<\alpha_{0}$, by (c), we get $\left|X^{-1} g_{0} \cap H_{\gamma}^{\alpha_{0}}\right|<\kappa$ for any $\gamma<\delta_{\alpha_{0}}$. Thus, again $X^{-1} g_{0}$ is unbounded in $\mathcal{H}^{\alpha_{0}}$ which proves the claim.

To carry out our construction of the increasing sequence of towers $\mathcal{H}^{\alpha}$, selectors $S_{\alpha}$ for $G / H^{\alpha}$, and ideals $\mathcal{N}_{\alpha}, \alpha<\rho$, fulfilling (a)-(d) and to make sure that the family $\mathcal{F}$ we produce satisfies point (i) of the theorem, we split our argument into two cases. We fix now for the rest of the proof an acceptable ideal $\mathcal{N}$ of subsets of $\kappa$. There are many choices for $\mathcal{N}$. For instance, the ideal of nonstationary subsets of $\kappa$ works. The referee suggested the following simple example. Consider the $\kappa$ complete ideal $\mathcal{N}^{\prime}$ on $\kappa \times \kappa$ generated by the graphs of functions from $\kappa$ to $\kappa$ and sets of the form $\{\alpha\} \times \kappa$ for $\alpha \in \kappa$. Let $\mathcal{N}$ be the ideal on $\kappa$ obtained by transferring $\mathcal{N}^{\prime}$ via a bijection between $\kappa$ and $\kappa \times \kappa$. Using regularity of $\kappa$ it is easy to check that this $\mathcal{N}$ is acceptable.

Case 1. $|G|=\kappa$

In this case, $\rho=1$ so we will have only one tower $\mathcal{H}^{0}$. This takes care of $(\mathrm{d})$. The height of the tower will be $\kappa$. We will need that $\mathcal{N}_{0}$ in addition to being acceptable is also not $\kappa$-saturated, that is, $\kappa$ can be partitioned into $\kappa$ many sets not in $\mathcal{N}_{0}$. Both ideals mentioned above as candidates for the ideal $\mathcal{N}$ are not $\kappa$-saturated so we can let $\mathcal{N}_{0}$ be the ideal $\mathcal{N}$ fixed above. Let $\left\langle B_{\alpha}: \gamma<\kappa\right\rangle$ list $\mathcal{I}$ in such a way that for each $B \in \mathcal{I}, B=B_{\gamma}$ on a set of $\gamma$ 's which is not in $\mathcal{N}_{0}$. This is possible 
since $|\mathcal{I}| \leq|G|=\kappa$. Pick $H_{\gamma}^{0}, \gamma<\kappa$, so that the sequence forms a $\kappa$-tower satisfying conditions (a)-(c) and additionally fulfilling

$$
H_{\alpha}^{0} \backslash\left(\bigcup_{\gamma<\alpha} H_{\gamma}^{0} \cup B_{\alpha}\right) \neq \emptyset
$$

Note that this can be easily done by transfinite recursion by letting $H_{\alpha}^{0}$ be the group generated by $\bigcup_{\gamma<\alpha} H_{\gamma}^{0}$, the $\alpha$ 'th element of $G$ in some fixed enumeration of $G$ in order type $\kappa$, and an element from $G \backslash\left(\bigcup_{\gamma<\alpha} H_{\gamma}^{0} \cup B_{\alpha}\right)$. This last set is nonempty since by induction one easily shows that $\left|\bigcup_{\gamma<\alpha} H_{\gamma}^{0}\right|<\kappa$ and by our assumption complements of members of $\mathcal{I}$ have cardinality $\kappa$. Pick $\{1\}$ as a selector $S_{0}$ for $G / H^{0}=G / G$. It remains to check that $\mathcal{F}=\mathcal{J}\left(\mathcal{H}^{0}, S_{0}, \mathcal{N}_{0}\right)$ satisfies (i). Let $X$ be a subset of $G$ with $|X|<\kappa$, and let $\mathcal{A}=\left\{S_{0}\left(A_{\gamma}\right): \gamma<\mu\right\}$ for some $\mu<\kappa$ and $A_{\gamma} \in \mathcal{N}_{0}$. Let $B$ be an element of $\mathcal{I}$. We show that $G \backslash X(\bigcup \mathcal{A})$ is not covered by $B$. Since $\kappa$ is regular, there exists $\gamma_{0} \in \kappa$ with $X \subseteq H_{\gamma_{0}}^{0}$. Since $\mathcal{N}_{0}$ is $\kappa$-complete and $\left\{\gamma<\kappa: B=B_{\gamma}\right\} \notin \mathcal{N}$, there exists $\gamma_{1}$ such that $B=B_{\gamma_{1}}, \gamma_{1}>\gamma_{0}$, and $\gamma_{1} \notin A$. Let $g_{0} \in G$ be an element of $H_{\gamma_{1}}^{0} \backslash\left(\bigcup_{\gamma<\gamma_{1}} H_{\gamma}^{0} \cup B_{\gamma_{1}}\right)$. Note further that $H_{\gamma_{1}}^{0} \backslash \bigcup_{\gamma<\gamma_{1}} H_{\gamma}^{0}$ is invariant with respect to translations by elements from $X^{-1}$ and, being a subset of $S_{0}(\kappa \backslash A)$, it is disjoint from $S_{0}(A)=\bigcup \mathcal{A}$. It therefore is disjoint from $X(\bigcup \mathcal{A})$. It follows that $g_{0}$ is not in $B \cup X(\bigcup \mathcal{A})$.

Case 2. $|G|>\kappa$

We first define an increasing sequence $\mathcal{H}^{\alpha}, \alpha<|G|$, of $\kappa$-towers of groups fulfilling conditions (a)-(c). This is accomplished by a recursive construction, on $\alpha<|G|$ and $\gamma<\kappa$, of groups $H_{\gamma}^{\alpha}$. Let $\mathcal{I}$ be listed as $\left\langle B_{\beta}: \beta<|G|\right\rangle$ in such a way that each $B \in \mathcal{I}$ is listed $|G|$ many times.

The following conditions are maintained in the construction:

(A) the $\alpha^{\prime}$ th element of $G$ in some fixed enumeration of $G$ in order type $|G|$ belongs to $H_{1}^{\alpha+1}$;

(B) if $\operatorname{cof}(\alpha) \neq \kappa$, then $H_{0}^{\alpha}=\bigcup_{\beta<\alpha} \bigcup_{\gamma<\kappa} H_{\gamma}^{\beta}$, also for any $1 \leq \gamma<\kappa, \bigcup_{\xi<\gamma} H_{\xi}^{\alpha} \subseteq$ $H_{\gamma}^{\alpha}$ and $0<\left|H_{\gamma}^{\alpha} / \bigcup_{\xi<\gamma} H_{\xi}^{\alpha}\right|<\kappa$ and finally $H_{0}^{0}=\{1\}$

(C) if $\operatorname{cof}(\alpha)=\kappa$, then there exists an increasing sequence $\beta_{\gamma}<\alpha, \gamma<\kappa$, cofinal in $\alpha$ with $H_{\gamma}^{\alpha}=\bigcup_{\xi<\kappa} H_{\xi}^{\beta_{\gamma}+1}$;

(D) $H_{1}^{\alpha+1} \backslash\left(B_{\alpha} \cup \bigcup_{\gamma<\kappa} H_{\gamma}^{\alpha}\right) \neq \emptyset$.

Put $\mathcal{H}^{\alpha}=\left\langle H_{\gamma}^{\alpha}: \gamma<\kappa\right\rangle, \alpha<|G|$. In (C) above, we have $\beta_{\gamma}+1$ in the definition of $H_{\gamma}^{\alpha}$ to make sure that $\bigcup_{\xi<\gamma} H_{\xi}^{\alpha} \neq H_{\gamma}^{\alpha}$. This condition is required by the definition of $\kappa$-tower.

Note that satisfying conditions $(\mathrm{A})-(\mathrm{C})$ presents no difficulty. (To achieve $(\mathrm{A})$ and (B) we use the assumption that if $H$ is a subgroup of $G$ and $a \in G$, then $\mid\langle H \cup\{a\}|<\kappa$.) Condition (D) is handled as follows. By induction we show that $\left|\bigcup_{\beta<\alpha} \bigcup_{\gamma<\kappa} H_{\gamma}^{\beta}\right| \leq \max (\kappa,|\alpha|)$. Since each element of $\mathcal{I}$ has complement of cardinality $|G|$ and, by our case assumption, $\max (\kappa,|\alpha|)<|G|$, we can always pick an element of $G$ outside of $B_{\alpha} \cup \bigcup_{\beta<\alpha} \bigcup_{\gamma<\kappa} H_{\gamma}^{\beta}$ and make sure that it ends up in $H_{1}^{\alpha+1}$ fulfilling (D).

Now we construct selectors $S_{\alpha}$ for $G / H^{\alpha}, \alpha<|G|$. We will write $H^{<\alpha}$ for $\bigcup_{\gamma<\alpha} H^{\gamma}$. Using (D) and the facts that the groups $H^{\alpha}$ increase as $\alpha$ increases and that $\left\langle B_{\beta}: \beta<|G|\right\rangle$ list each member of $\mathcal{I}|G|$ many times, we can find a set $E$ such 
that

$$
\left|E \cap\left(H^{\alpha} \backslash H^{<\alpha}\right)\right| \leq 1 \text { for } \alpha<|G|,
$$

and

$$
\left|E \backslash\left(B_{\beta} \cup H^{\alpha}\right)\right|=|G| \text { for any } \alpha, \beta<|G| .
$$

Using this last property, we apply transfinite recursion on triples $(\gamma, \beta, \alpha) \in \kappa \times$ $|G| \times|G|$ ordered in order type $|G|$ to partition $E$ into subsets $E_{\gamma}, \gamma<\kappa$, so that they fulfill

$$
E_{\gamma} \backslash\left(B_{\beta} \cup H^{\alpha}\right) \neq \emptyset \text { for any } \gamma<\kappa \text { and } \alpha, \beta<|G| .
$$

Note that this implies that

(3) $E_{\gamma} \backslash H^{\alpha}$ is not included in a member of $\mathcal{I}$ for any $\gamma<\kappa$ and $\alpha<|G|$.

Let $T_{\alpha}, \alpha<|G|$, be a selector for $\left(H^{\alpha} \backslash H^{<\alpha}\right) / H^{<\alpha}$ such that $E \cap\left(H^{\alpha} \backslash H^{<\alpha}\right) \subseteq$ $T_{\alpha}$. This is possible by (2).

Now, fix $\alpha_{0}<|G|$. To define $S_{\alpha_{0}}$, the desired selector for $G / H^{\alpha_{0}}$, we will need two more ingredients. First, we define a sequence of sets $T^{\alpha} \subseteq G, \alpha_{0} \leq \alpha<|G|$, by the formulas:

$$
\begin{gathered}
T^{\alpha_{0}}=\{1\} \\
T^{\alpha}=\left(\bigcup_{\alpha_{0} \leq \gamma<\alpha} T^{\gamma}\right) T_{\alpha} \text { for } \alpha>\alpha_{0} .
\end{gathered}
$$

Note that for $\alpha>\alpha_{0}, T^{\alpha}$ is a selector for $\left(H^{\alpha} \backslash H^{<\alpha}\right) / H^{\alpha_{0}}$. It follows from this that $\bigcup_{\alpha_{0} \leq \alpha<|G|} T^{\alpha}$ is a selector for $G / H^{\alpha_{0}}$. Second, we pick $f_{\alpha} \in H^{\alpha_{0}}$, for $\alpha<|G|$, as follows:

if $E \cap\left(H^{\alpha} \backslash H^{<\alpha}\right)=\emptyset$, let $f_{\alpha}=1$;

if $E \cap\left(H^{\alpha} \backslash H^{<\alpha}\right) \neq \emptyset$, then by the fact that the $E_{\gamma}$ 's partition $E$ and by (2), there is a unique $\gamma<\kappa, E_{\gamma} \cap\left(H^{\alpha} \backslash H^{<\alpha}\right) \neq \emptyset$; pick this $\gamma$ and let $f_{\alpha}$ be an arbitrary element of $H_{\gamma}^{\alpha_{0}} \backslash \bigcup_{\xi<\gamma} H_{\xi}^{\alpha_{0}}$.

Note that even though it is not reflected in our notation, the sequence $\left\langle f_{\alpha}: \alpha<|G|\right\rangle$ depends on $\alpha_{0}$ as do the $T^{\alpha}$ 's. We put

$$
S_{\alpha_{0}}=\bigcup_{\alpha_{0} \leq \alpha<|G|} f_{\alpha} T^{\alpha} .
$$

Since each $T^{\alpha}, \alpha>\alpha_{0}$, is a selector for $\left(H^{\alpha} \backslash H^{<\alpha}\right) / H^{\alpha_{0}}, S_{\alpha_{0}}$ is a selector for $G / H^{\alpha_{0}}$.

Finally, for each $\alpha<|G|$, let $\mathcal{N}_{\alpha}$ be equal to the fixed acceptable ideal $\mathcal{N}$ of subsets of $\kappa$.

Claim 3. Let $S_{\alpha_{\gamma}}\left(A_{\gamma}\right) \in \mathcal{F}$ and $g_{\gamma} \in G, \gamma<\mu<\min (\kappa, \operatorname{cof}(|G|))$. There exist $\bar{\alpha}<|G|$ such that the complement of

$$
\left\{\xi<\kappa: G \backslash \bigcup_{\gamma<\mu} g_{\gamma} S_{\alpha_{\gamma}}\left(A_{\gamma}\right) \supseteq E_{\xi} \backslash H^{\bar{\alpha}}\right\}
$$

is in $\mathcal{N}$.

Proof. By $\kappa$-completeness of $\mathcal{N}$ and the fact that the sequence of groups $H^{\alpha}$, $\alpha<|G|$, is increasing, it will suffice to show that for any $A \in \mathcal{N}$, any $g \in G$, and 
any $\alpha_{0}<|G|$, there exists $\alpha_{1}<|G|$ such that the complement in $\kappa$ of $\{\xi<\kappa$ : $\left.G \backslash g S_{\alpha_{0}}(A) \supseteq E_{\xi} \backslash H^{\alpha_{1}}\right\}$ is in $\mathcal{N}$.

So we fix $A \in \mathcal{N}, g \in G$, and $\alpha_{0}<|G|$. The above construction of $S_{\alpha_{0}}$ produces the $T^{\alpha}$ 's, $\alpha_{0} \leq \alpha<|G|$, and the $f_{\alpha}$ 's, $\alpha<|G|$. We will need these objects in what follows. Since $\bigcup_{\alpha_{0} \leq \alpha<|G|} T^{\alpha}$ is a selector for $G / H^{\alpha_{0}}$,

$$
g^{-1}=h t
$$

for unique $h \in H^{\alpha_{0}}$ and $t \in \bigcup_{\alpha_{0} \leq \alpha<|G|} T^{\alpha}$. Let

$$
\alpha_{1}=\min \left\{\alpha<|G|: g \in H^{\alpha}\right\} \text { and } \gamma_{0}=\min \left\{\gamma<\kappa: h \in H_{\gamma}^{\alpha_{0}}\right\} .
$$

We claim that, for any $\gamma \in \kappa \backslash A$ with $\gamma>\gamma_{0}$, we have $G \backslash g S_{\alpha_{0}}(A) \supseteq E_{\gamma} \backslash H^{\alpha_{1}}$; this will be enough since $A \in \mathcal{N}$. Assume towards a contradiction that there exists $s$ such that, for some $\gamma_{1}>\gamma_{0}, \gamma_{1} \in \kappa \backslash A$,

$$
s \in E_{\gamma_{1}}, s \notin H^{\alpha_{1}} \text {, and } s \in g S_{\alpha_{0}}(A) \text {. }
$$

By (A), we can find $\alpha_{2}<|G|$ such that $s \in H^{\alpha_{2}} \backslash H^{<\alpha_{2}}$. Clearly $\alpha_{1}<\alpha_{2}$, so

$$
g^{-1} \in H^{<\alpha_{2}} .
$$

Note that since $s \in H^{\alpha_{2}} \backslash H^{<\alpha_{2}}$ and, by (5), $s \in E_{\gamma_{1}}$, we see that $\gamma_{1}$ is the unique $\gamma<\kappa$ with $E_{\gamma} \cap\left(H^{\alpha_{2}} \backslash H^{<\alpha_{2}}\right) \neq \emptyset$. Since additionally $\gamma_{1} \notin A \cup\left(\gamma_{0}+1\right)$, by our choice of $f_{\alpha}$ 's for $\alpha_{0}$, we get

$$
f_{\alpha_{2}} \notin \bigcup_{\gamma \in A}\left(H_{\gamma}^{\alpha_{0}} \backslash \bigcup_{\xi<\gamma} H_{\xi}^{\alpha_{0}}\right) \cup H_{\gamma_{0}}^{\alpha_{0}} .
$$

We will contradict (7) by computing $g^{-1}$ in a way different from (4). By (5), $g^{-1} s \in S_{\alpha_{0}}(A)$, so

$$
g^{-1}=h_{1} f_{\alpha_{2}} t_{1} t_{2} s^{-1}
$$

where $h_{1} \in \bigcup_{\gamma \in A}\left(H_{\gamma}^{\alpha_{0}} \backslash \bigcup_{\xi<\gamma} H_{\xi}^{\alpha_{0}}\right), t_{1} \in \bigcup_{\alpha_{0} \leq \gamma<\alpha_{2}} T^{\gamma}, t_{2} \in T_{\alpha_{2}}$. Since $s \in$ $E \cap\left(H^{\alpha_{2}} \backslash H^{<\alpha_{2}}\right)$, we have $s \in T_{\alpha_{2}}$. From (8) and (6), we see that $t_{2} s^{-1}=$ $\left(h_{1} f_{\alpha_{2}} t_{1}\right)^{-1} g^{-1} \in H^{<\alpha_{2}}$. It follows therefore that $t_{2}=s$. Combining this with (8), we obtain

$$
g^{-1}=h_{1} f_{\alpha_{2}} t_{1} .
$$

Note now that $h_{1} f_{\alpha_{2}} \in H^{\alpha_{0}}$ and $t_{1} \in \bigcup_{\alpha_{0} \leq \alpha<\alpha_{2}} T^{\alpha}$. Comparing (9) with (4), by uniqueness of representation of $g^{-1}$ as the product of an element from $H^{\alpha_{0}}$ and an element of the selector $\bigcup_{\alpha_{0} \leq \alpha<|G|} T^{\alpha}$, we get $h=h_{1} f_{\alpha_{2}}$ (and $t=t_{1}$ ). Thus, $h_{1} f_{\alpha_{2}} \in H_{\gamma_{0}}^{\alpha_{0}}$, whence

$$
f_{\alpha_{2}} \in h_{1}^{-1} H_{\gamma_{0}}^{\alpha_{0}} \subseteq \bigcup_{\gamma \in A}\left(H_{\gamma}^{\alpha_{0}} \backslash \bigcup_{\xi<\gamma} H_{\xi}^{\alpha_{0}}\right) H_{\gamma_{0}}^{\alpha_{0}} \subseteq \bigcup_{\gamma \in A}\left(H_{\gamma}^{\alpha_{0}} \backslash \bigcup_{\xi<\gamma} H_{\xi}^{\alpha_{0}}\right) \cup H_{\gamma_{0}}^{\alpha_{0}} .
$$

This, however, contradicts (7) proving the claim. 
We will now consider two subcases. In either of them we will specify an ordinal $\rho$ and $\mathcal{H}^{\alpha}, S_{\alpha}, \mathcal{N}_{\alpha}, \alpha<\rho$, and we will check that $\mathcal{F}$ defined as in (0) fulfills condition (i) and conditions (a)-(d) (which will ensure (ii) and (iii) according to Claims 1 and 2).

Subcase 1. $\operatorname{cof}(|G|) \neq \kappa$.

In this case, we let our increasing sequence of towers be $\mathcal{H}^{\alpha}, \alpha<|G|$, constructed above. So $\rho=|G|$. Note that (a)-(c) follow immediately from (A)-(C) and, by the subcase assumption, condition (d) is fulfilled as well. It remains to check (i). Let $\mu<\min (\kappa, \operatorname{cof}(|G|))$. Let $g_{\gamma} \in G, S_{\alpha_{\gamma}}\left(A_{\gamma}\right) \in \mathcal{F}$ for $\alpha_{\gamma}<\rho, \gamma<\mu$. From Claim 3 and (3), we get that $G \backslash \bigcup_{\gamma<\mu} g_{\gamma} S_{\alpha_{\gamma}}\left(A_{\gamma}\right)$ is not included in an element of $\mathcal{I}$.

Subcase 2. $\operatorname{cof}(|G|)=\kappa$.

In this case, in addition to the towers $\mathcal{H}^{\alpha}, \alpha<|G|$, constructed above, we will need to define one more tower of groups, that is, $\rho$ will be $|G|+1$. This will take care of condition (d). Put $|G|=\lambda$. Define $\mathcal{H}^{\lambda}$ to be the sequence $\left\langle H_{\alpha}^{\lambda}: \alpha<\lambda\right\rangle$ where

$$
H_{\alpha}^{\lambda}=H^{\alpha+1}\left(=\bigcup \mathcal{H}^{\alpha+1}\right) .
$$

Note that this definition along with (A)-(C) implies conditions (a)-(c). Let $S_{\lambda}=$ $\{1\}$. Now it remains to define a $\kappa$-complete ideal $\mathcal{N}_{\lambda}$ on $\lambda$. We do it as follows. For $\gamma<\kappa$, let

$$
D_{\gamma}=\left\{\alpha \in \lambda: E_{\gamma} \cap\left(H^{\alpha} \backslash H^{<\alpha}\right) \neq \emptyset\right\}
$$

for $f \in \lambda^{\kappa}$, let

$$
B_{f}=\left\{\alpha \in \lambda: \exists \gamma \in \kappa \alpha=f(\gamma) \text { and } E_{\gamma} \cap\left(H^{\alpha} \backslash H^{<\alpha}\right) \neq \emptyset\right\} \text {, }
$$

and

$$
C=\left\{\alpha \in \lambda: \forall \gamma<\kappa E_{\gamma} \cap\left(H^{\alpha} \backslash H^{<\alpha}\right)=\emptyset\right\} .
$$

Define $\mathcal{N}_{\lambda}$ to be the $\kappa$-complete ideal generated by the sets $D_{\gamma}, \gamma<\kappa, B_{f}, f \in \lambda^{\kappa}$, and $C$. We need to check acceptability of $\mathcal{N}_{\lambda}$.

First we need to see that $\lambda \backslash A$ is cofinal in $\lambda$ for any $A \in \mathcal{N}_{\lambda}$. Let $\alpha_{0} \in \lambda$ and consider $\left\{D_{\gamma}: \gamma \in a\right\}$ and $\left\{B_{f}: f \in b\right\}$ with $|a|,|b|<\kappa$. Pick $\gamma_{0} \in \kappa \backslash a$. Note that $\left|E_{\gamma_{0}}\right|=\lambda$, so by (2), $\left\{\alpha<\lambda: E_{\gamma_{0}} \cap\left(H^{\alpha} \backslash H^{<\alpha}\right) \neq \emptyset\right\}$ is cofinal in $\lambda$. This allows us to pick $\alpha^{\prime}$ from this set with $\alpha^{\prime}>\alpha_{0}$ and $\alpha^{\prime}>\sup \left\{f\left(\gamma_{0}\right): f \in b\right\}$. By (2) and disjointness of the $E_{\gamma}$ 's this $\alpha^{\prime}$ does not belong to $\bigcup_{\gamma \in a} D_{\gamma} \cup \bigcup_{f \in b} B_{f} \cup C$.

$\kappa$-completeness being obvious, to see that $\mathcal{N}_{\lambda}$ is acceptable, it remains to show that for any $B \subseteq \lambda$ cofinal in $\lambda$ there exists $A \in \mathcal{N}_{\lambda}$ cofinal in $\lambda$ with $A \subseteq B$. Since $\operatorname{cof}(\lambda)=\kappa$, we can assume that $|B|=\kappa$ and that each subset of $B$ of cardinality $\kappa$ is cofinal in $\lambda$. Assume that $C \cap B$ and $D_{\gamma} \cap B$ are not cofinal in $\lambda$ for all $\gamma<\kappa$. (Otherwise, we are done.) This means that $|C \cap B|<\kappa$ and $\left|D_{\gamma} \cap B\right|<\kappa$ for all $\gamma<\kappa$. It follows, by regularity of $\kappa$, that the set $a=\left\{\gamma<\kappa: D_{\gamma} \cap B \neq \emptyset\right\}$ has cardinality $\kappa$. We pick now a function $f: \kappa \rightarrow \lambda$ such that $f(\gamma) \in D_{\gamma} \cap B$ for $\gamma \in a$. Clearly $B_{f} \cap B$ has cardinality $\kappa$ and so is cofinal in $\lambda$.

To finish the proof of the theorem, it remains to see that (i) holds. Fix $\mu<\kappa$ and $g_{\gamma} S_{\alpha_{\gamma}}\left(A_{\gamma}\right)$, with $\alpha_{\gamma}<\lambda$, and $g_{\gamma}^{\prime} S_{\lambda}\left(A_{\gamma}^{\prime}\right)$ for $\gamma<\mu$ where $g_{\gamma}, g_{\gamma}^{\prime} \in G, A_{\gamma} \in \mathcal{N}$, and $A_{\gamma}^{\prime} \in \mathcal{N}_{\lambda}$. We will assume, without loss of generality, that for each $\gamma<\mu$, $A_{\gamma}^{\prime}=D_{\eta_{\gamma}}$ for some $\eta_{\gamma}<\kappa$, or $A_{\gamma}^{\prime}=B_{f_{\gamma}}$ for some $f_{\gamma} \in \lambda^{\kappa}$, or $A_{\gamma}^{\prime}=C$. In fact, we assume that these three possibilities are realized according to whether $\gamma \in X_{1}$, 
$\gamma \in X_{2}, \gamma \in X_{3}$ with $X_{1} \cup X_{2} \cup X_{3}=\mu$. Now, using Claim 3, we find $\bar{\alpha}<\lambda$ and $A \in \mathcal{N}$ such that

$$
G \backslash \bigcup_{\gamma<\mu} g_{\gamma} S_{\alpha_{\gamma}}\left(A_{\gamma}\right) \supseteq E_{\xi} \backslash H^{\bar{\alpha}} \text { for } \xi \in \kappa \backslash A .
$$

Fix $\alpha^{\prime}<\lambda$ with $g_{\gamma}^{\prime} \in H^{\alpha^{\prime}}$ for all $\gamma<\mu$. Then

$$
g_{\gamma}^{\prime} S_{\lambda}\left(A_{\gamma}^{\prime}\right) \backslash H^{\alpha^{\prime}}=g_{\gamma}^{\prime} \bigcup_{\alpha \in A_{\gamma}^{\prime}}\left(H^{\alpha} \backslash H^{<\alpha}\right) \backslash H^{\alpha^{\prime}}=\bigcup_{\alpha \in A_{\gamma}^{\prime}, \alpha>\alpha^{\prime}}\left(H^{\alpha} \backslash H^{<\alpha}\right) .
$$

If $\gamma \in X_{1}$, this gives

$$
g_{\gamma}^{\prime} S_{\lambda}\left(A_{\gamma}^{\prime}\right) \backslash H^{\alpha^{\prime}}=\bigcup\left\{H^{\alpha} \backslash H^{<\alpha}: E_{\eta_{\gamma}} \cap\left(H^{\alpha} \backslash H^{<\alpha}\right) \neq \emptyset, \alpha>\alpha^{\prime}\right\} .
$$

Now using the fact that $\kappa \backslash A$ has cardinality $\kappa$, pick $\bar{\xi} \in \kappa$ so that

$$
\bar{\xi} \notin A,
$$

and $\bar{\xi} \neq \eta_{\gamma}$ for all $\gamma<\mu$. This, (2), disjointness of the $E_{\xi}$ 's, and (12) imply that

$$
G \backslash \bigcup_{\gamma \in X_{1}} g_{\gamma}^{\prime} S_{\lambda}\left(A_{\gamma}^{\prime}\right) \supseteq E_{\bar{\xi}} \backslash H^{\alpha^{\prime}} .
$$

There exists $\alpha^{\prime \prime}<\lambda$ such that $f_{\gamma}(\bar{\xi})<\alpha^{\prime \prime}$ for $\gamma \in X_{2}$. Making $\alpha^{\prime \prime}>\alpha^{\prime}$ and using (11), we get from this

$$
G \backslash \bigcup_{\gamma \in X_{2}} g_{\gamma}^{\prime} S_{\lambda}\left(A_{\gamma}^{\prime}\right) \supseteq E_{\bar{\xi}} \backslash H^{\alpha^{\prime \prime}}
$$

Using (11) we obviously also have

$$
G \backslash \bigcup_{\gamma \in X_{3}} g_{\gamma}^{\prime} S_{\lambda}(C) \supseteq E_{\bar{\xi}} \backslash H^{\alpha^{\prime}} .
$$

Now it follows from (10) and (13)-(16) that

$$
G \backslash\left(\bigcup_{\gamma<\mu} g_{\gamma} S_{\alpha_{\gamma}}\left(A_{\gamma}\right) \cup \bigcup_{\gamma<\mu} g_{\gamma}^{\prime} S_{\lambda}\left(A_{\gamma}^{\prime}\right)\right) \supseteq E_{\bar{\xi}} \backslash H^{\alpha^{\prime \prime \prime}}
$$

where $\alpha^{\prime \prime \prime}=\max \left(\bar{\alpha}, \alpha^{\prime \prime}\right)$. By (3), this means that the set on the left hand side cannot be covered by an element of $\mathcal{I}$ and (i) is proved.

\section{Extending HaAr measures}

Results in this section contribute to the program of investigating nonmeasurability in the context of translation invariant measures on groups. The recent book by Kharazishvili [9] is an up-to-date introduction to the subject.

We will need a few definitions. Let $\mu$ be a left translation invariant measure defined on a $\sigma$-algebra of subsets of a group $G$. Sets in the $\sigma$-algebra are called 
$\mu$-measurable. All measures are $\sigma$-additive and are not identically equal to zero. Without loss of generality, we also assume that each subset of a measure zero set is measurable (and has measure zero). A family $\mathcal{B}$ of subsets of $G$ is said to be cofinal in $\mu$ if each $\mu$-measurable set of positive measure contains a set from $\mathcal{B}$. The measure $\mu$ is called $\sigma$-finite if $G$ is a countable union of $\mu$-measurable sets of finite measure. We say that $\mu$ is metrically transitive if for any two $\mu$-measurable sets $A$ and $B$ of positive measure there exists a $g \in G$ with $\mu(A \cap g B)>0$.

Vitali's construction produces a set which is nonmeasurable with respect to any invariant extension of Lebesgue measure as a selector of a dense countable subgroup of the reals. In [14, Theorem 1], it was shown that if $G$ is an uncountable group, then for an arbitrary left translation invariant $\sigma$-finite measure $\mu$ on $G$ one can find a countable subgroup of $G$ each of whose selectors is nonmeasurable with respect to any left translation invariant extension of $\mu$. This result naturally raises the question of whether selectors of uncountable subgroups can be made measurable with respect to invariant measures. Note that the matter is delicate since, as proved by Erdős and Mauldin and Kharazishvili (see the proofs of the main results in [5] and [8]), for each measure $\mu$ as above and each subgroup $H$ of $G$ of cardinality $\aleph_{1}$ and each selector $S$ of $G / H$ there exists a family of left translates of $S$ by elements of $H$ whose union is not $\mu$-measurable. (Note that left translates by elements of $H$ of a selector of $G / H$ are again selectors of $G / H$.) As a partial answer to the above question, it was proved in [14, Theorem 2] that if $\operatorname{cof}(|G|)>$ $\aleph_{0}$ and $\mu$ is $\sigma$-finite and metrically transitive, then it admits a left translation invariant extension which measures a selector of each subgroup of cardinality $|G|$. In particular, if the Continuum Hypothesis holds, then the left Haar measure on any locally compact, second countable group $G$ admits a left translation invariant extension which measures at least one selector of any uncountable subgroup. Nowik in [11] showed that in the case when $G$ is equal to $\mathbb{R}$, the additive group of the reals, the conclusion follows from the negation of the Continuum Hypothesis thus eliminating the Continuum Hypothesis from the assumptions in the case $G=\mathbb{R}$. Corollary 2.2 below extends this result to all locally compact, second countable, Abelian groups. Corollary 2.1, of which 2.2 is a consequence, shows that, apart from $\operatorname{cof}(|G|)>\aleph_{0}$, only an assumption on cofinal in $\mu$ families is needed for the existence of the desired extension. Both these corollaries indicate that particular algebraic properties of the group $G$ are of secondary importance for the existence of such invariant extensions. (For more background on translation invariant extensions of translation invariant measures see [9], and for different aspects of measurability of selectors of subgroups of $\mathbb{R}$ see [3].)

Corollary 2.1. Let $G$ be an Abelian group with a translation invariant measure $\mu$. Assume that $\operatorname{cof}(|G|)>\aleph_{0}$ and that there exists a cofinal in $\mu$ family of cardinality $|G|$ of sets of cardinality $|G|$. Then there exists a measure $\nu$ on $G$ such that

(i) $\nu$ is a translation invariant extension of $\mu$;

(ii) for any uncountable $X \subseteq G$ there exists $A \subseteq G$ with $\nu(A)=0$ and $X+A=G$; in particular, each uncountable subgroup of $G$ has a $\nu$-measurable selector.

Proof. Let $\mathcal{B}$ be a family of subsets of $G$ cofinal in $\mu$ as in the statement of the corollary. Let $\mathcal{I}=\{G \backslash X: X \in \mathcal{B}\}$. Let $\mathcal{F}$ be the family produced in Theorem 1.1 for $\mathcal{I}$ and $\kappa=\aleph_{1}$. Let $\mathcal{J}$ be the translation invariant $\sigma$-ideal generated by $\mathcal{F}$ and the family of all $\mu$-measure zero sets. Note first that each element of $\mathcal{J}$ has inner measure zero. Otherwise, some set of the form $A \cup(X+(\cup \mathcal{A}))$ has positive 
inner measure where $A$ is of measure zero and $X \subseteq G$ and $\mathcal{A} \subseteq \mathcal{F}$ are countable. It follows that $X+(\bigcup \mathcal{A})$ has positive inner measure and, therefore, contains a set from $\mathcal{B}$. But this is prohibited by Theorem 1.1(i) (we use here $\operatorname{cof}(|G|)>\aleph_{0}$ ) and our definition of $\mathcal{I}$. Now, it follows from Szpilrajn's lemma [15, Section 2] that there exists $\nu$ as in (i) such that the family of $\nu$-measure zero sets contains $\mathcal{J}$. Thus, (ii) is guaranteed by Theorem 1.1(ii).

Remark. In the context of Theorem 2 from [14], it would be interesting to see if the assumption on the existence of the cofinal family for $\mu$ in Corollary 2.1 can be replaced by $\sigma$-finiteness of $\mu$ (or by $\sigma$-finiteness and metric transitivity). Here it may be worth pointing out that for the above proof to work, we can weaken the condition "each $\mu$-measurable set of positive measure contains a set from $\mathcal{B}$ " in the definition of cofinality in $\mu$ to the condition "for each $\mu$-measurable set $A$ of positive measure there exists a countable set $D \subseteq G$ such that $D+A$ contains a set from $\mathcal{B}$." The modification of the proof is straightforward.

Corollary 2.2. Let $G$ be a locally compact, second countable, Abelian group. Then there exists a measure $\nu$ on $G$ such that

(i) $\nu$ is a translation invariant extension of the Haar measure;

(ii) for any uncountable $X \subseteq G$ there exists $A \subseteq G$ with $\nu(A)=0$ and $X+A=G$; in particular, each uncountable subgroup of $G$ has a $\nu$-measurable selector.

Proof. Clearly all the assumptions of Corollary 2.1 are fulfilled here: $\operatorname{cof}(|G|)=$ $\operatorname{cof}\left(2^{\aleph_{0}}\right)>\aleph_{0}$ and the family of all Borel sets of positive Haar measure is cofinal in Haar measure, is of cardinality $2^{\aleph_{0}}$, and consists of sets of cardinality $2^{\aleph_{0}}$.

Questions related to the above result were studied by Cichon, Kharazishvili, and Wȩglorz in [3]. Among other things they proved [3, Theorem 6] that if Martin's Axiom (MA) holds, then there is a subgroup of $\mathbb{R}$ of cardinality $2^{\aleph_{0}}$ none of whose selectors is Lebesgue measurable (i.e., is of Lebesgue measure zero) and there is another subgroup of cardinality $2^{\aleph_{0}}$ none of whose selectors has the Baire property (i.e., is meager). Actually, only the first part of the above statement is proved there explicitly but the method extends easily to the Baire property case. Jacek Cichon asked (private communication) if it is possible to construct, under MA, one group of cardinality $2^{\aleph_{0}}$ with both of these properties, i.e., none of the selectors of this group should be Lebesgue measurable or have the Baire property. In Theorem 2.4, we show that a modification of a construction due to Friedman and Talagrand [6] and Erdős, Kunen, and Mauldin [4] gives an affirmative answer to this question.

Friedman and Talagrand and, independently, Erdős, Kunen, and Mauldin constructed, assuming MA, the following subset $A$ of $\mathbb{R}$. Let $\phi: \mathcal{P}(\mathbb{N}) \rightarrow \mathbb{R}$ be defined by $\phi(M)=\sum_{i \in M} 2^{-i}$. Define $A$ to be $\left\{\phi\left(M_{\alpha}\right): \alpha<2^{\aleph_{0}}\right\}$ where $\left\{M_{\alpha}: \alpha<2^{\aleph_{0}}\right\}$ is a family of infinite subsets of $\mathbb{N}$ such that

(a) $M_{\beta} \backslash M_{\alpha}$ is finite whenever $\alpha<\beta$;

(b) $\forall\left(m_{n}\right) \in \mathbb{N}^{\mathbb{N}} \exists \alpha<2^{\aleph_{0}} M_{\alpha}$ dominates $\left(m_{n}\right)$.

(Here and below $M$ dominates $\left(m_{n}\right)$ means that the $n$ 'th element of $M$ is greater than or equal to $m_{n}$.)

Such a family exists if MA is assumed. It was proved that if $\lambda(X)=0$, then $\lambda(A+X)=0$ ([4] and [6]), and if $X$ is meager, then $A+X$ is meager as well ([6]). Modifying the proof from [6], we show here that the group generated by $A$ has the same property. 
Let $B_{M}=\{\phi(N): N \subseteq M\}, M \subseteq \mathbb{N}$, and let $\langle C\rangle$ stand for the group generated by a set $C \subseteq \mathbb{R}$.

\section{Lemma 2.3.}

(i) For any $X$ with $\lambda(X)=0$ there is a sequence $\left(m_{n}\right) \in \mathbb{N}^{\mathbb{N}}$ such that if $M$ dominates $\left(m_{n}\right)$, then $\lambda\left(X+\left\langle B_{M}\right\rangle\right)=0$.

(ii) Similarly for $X$ meager.

Proof. We give an argument for (i) only. It is a slight modification of the proof from [6] and is included here to indicate the needed changes. Changes needed to prove (ii) are similar and the proof of (ii) is left to the reader willing to consult [6]. Alternatively one can apply Shelah's theorem [13], see also [1, Theorem 2.7.20], which makes (ii) a consequence of (i).

If $\lambda(X)=0$, we can find a sequence $\left(U_{n}\right)$ such that each $U_{n}$ is a finite union of open intervals, $X \subseteq \bigcap_{m} \bigcup_{n>m} U_{n}$, and $\sum_{n} 2^{n^{2}} \lambda\left(U_{n}\right)<\infty$. Choose $m_{n}$ so that $\lambda\left(I+U_{n}\right) \leq 2 \lambda\left(U_{n}\right)$ for any interval $\mathcal{N}$ with $\lambda(I) \leq n 2^{1-m_{n}}$. Let $M$ dominate $\left(m_{n}\right)$. It is easy to see that there are $H_{n} \subseteq \mathbb{R},\left|H_{n}\right| \leq 2^{n}, n \in \mathbb{N}$, with $B_{M} \subseteq$ $H_{n}+\left[0,2^{1-m_{n}}\right]$. Let $\epsilon_{0}, \ldots, \epsilon_{k} \in\{-1,0,1\}$. Then for $r \geq k$, we have

$$
X+\sum_{i=0}^{k} \epsilon_{i} B_{M} \subseteq \bigcup_{n \geq r}\left(\sum_{i=0}^{k} \epsilon_{i} H_{n}+\sum_{i=0}^{k} \epsilon_{i}\left[0,2^{1-m_{n}}\right]+U_{n}\right)=\bigcup_{n \geq r} H+I+U_{n},
$$

where $|H| \leq 2^{k n} \leq 2^{n^{2}}$ and $\lambda(I) \leq k 2^{1-m_{n}} \leq n 2^{1-m_{n}}$. Thus, by the choice of $\left(m_{n}\right)$ and $U_{n}$, we get

$$
\lambda\left(X+\sum_{i=0}^{k} \epsilon_{i} B_{M}\right) \leq \sum_{n=r}^{\infty} 2^{n^{2}} \lambda\left(I+U_{n}\right) \leq \sum_{n=r}^{\infty} 2^{n^{2}+1} \lambda\left(U_{n}\right) \rightarrow 0 \text { as } r \rightarrow \infty,
$$

i.e., $\lambda\left(X+\sum_{i=0}^{k} \epsilon_{i} B_{M}\right)=0$. It follows that $\lambda\left(X+\left\langle B_{M}\right\rangle\right)=0$.

Now we have the following theorem.

Theorem 2.4. Assume $M A$. There exists a group $G \subseteq \mathbb{R}$ with $|G|=2^{\aleph_{0}}$ such that $G+X$ is of Lebesgue measure zero if $X$ is, and $G+X$ is meager if $X$ is; in particular, none of the selectors of $\mathbb{R} / G$ is Lebesgue measurable or has the Baire property.

Proof. To get the "in particular" part one only needs to notice that if $G$ has a measurable selector, then it must have measure zero, and similarly each selector with Baire property must be meager.

It is easy to see that, for all $\alpha<2^{\aleph_{0}},\left|A \backslash\left(\mathbb{Q}+B_{M_{\alpha}}\right)\right|<2^{\aleph_{0}}$ where $\mathbb{Q}$ stands for the rationals. It follows from this that for any $\alpha<2^{\aleph_{0}}$ there is a group $H \subseteq \mathbb{R}$ with $|H|<2^{\aleph_{0}}$ and $\langle A\rangle \subseteq H+\left\langle B_{M_{\alpha}}\right\rangle$. Thus, to show that given $X \subseteq \mathbb{R}$ with $\lambda(X)=0$ (X meager) we have $\lambda(X+\langle A\rangle)=0(X+\langle A\rangle$ meager, respectively $)$, it is enough to find $\alpha<2^{\aleph_{0}}$ such that $\lambda\left(X+\left\langle B_{M_{\alpha}}\right\rangle\right)=0\left(X+\left\langle B_{M_{\alpha}}\right\rangle\right.$ meager, respectively). Remember that we assume MA which implies that the union of $<2^{\aleph_{0}}$ sets of Lebesgue measure zero is of measure zero and the same holds for meager sets. The existence of such an $\alpha$ is guaranteed by Lemma 2.3.

Remark. An analysis of the proof of the above theorem reveals that the theorem can be deduced merely from some consequences of MA. To obtain precisely the 
statements below, we use some well-known results concerning Cichon's diagram which can be found in [1, Chapter 2]. To get, with the argument above, a subgroup $G$ of $\mathbb{R}$ of cardinality $2^{\aleph_{0}}$ such that, for any set $X$ of Lebesgue measure zero, $G+X$ has Lebesgue measure zero and, any meager set $X, G+X$ is meager, one only needs to assume that the additivity of the ideal of Lebesgue measure zero sets is $2^{\aleph_{0}}$. Note that a subgroup $G$ of cardinality $2^{\aleph_{0}}$ will not have selectors which are Lebesgue measurable or have the Baire property if only $G+X \neq \mathbb{R}$ for any $X$ which is meager or has Lebesgue measure zero. The construction above achieves it with a slightly weaker assumption that the additivity of the meager ideal and the covering of the measure zero ideal are both $2^{\aleph_{0}}$.

\section{Translating Sets into COMPlements}

In [12], Seredyński studied the following operation. Let $\mathcal{J}$ be a family of subsets of an Abelian group $G$. Define $\mathcal{J}^{*}$ to be the family of all $A \subseteq G$ such that any set in $\mathcal{J}$ can be translated into the complement of $A$, that is,

$$
\mathcal{J}^{*}=\{X \subseteq G: \forall A \in \mathcal{J} \exists g \in G(X+g) \cap A=\emptyset\} .
$$

The definition and investigation of the operation * were motivated by the result of Galvin, Mycielski, and Solovay that, for $G$ the additive group of the reals, \{meager sets $^{*}=\{$ strong measure zero sets $\}$. Seredyński asked in [12, p. 219, P1368] if there exists (in ZFC) a family of subsets of $\mathbb{R}$ such that $\mathcal{J}^{*}=\{$ countable subsets of $\mathbb{R}\} .{ }^{1}$ It was noticed in [12, p. 209 and p. 212] that the following consistency results follow from the above mentioned Galvin-Mycielski-Solovay theorem and results of Laver and Carlson:

(a) $\{\text { meager sets }\}^{*}=\{$ countable sets $\}$ provided the Borel Conjecture holds by [7], which is consistent by [10];

(b) $\{\text { Lebesgue measure zero sets }\}^{*}=\{$ countable sets $\}$ is the dual Borel conjecture which is consistent by [2]

The corollary below answers Seredyński's question in the affirmative. In fact, since $\operatorname{cof}\left(2^{\aleph_{0}}\right) \geq \aleph_{1}$, we get a translation invariant $\sigma$-ideal $\mathcal{J}$ of subsets of $\mathbb{R}$ for which $\mathcal{J}^{*}=\{$ countable subsets of $\mathbb{R}\}$.

Corollary 3.1. Let $G$ be an Abelian group, and let $\kappa$ be a regular cardinal. There is a translation invariant ideal $\mathcal{J}$ on $G$ such that $\mathcal{J}^{*}=\{X \subseteq G:|X|<\kappa\}$. If, additionally, $\operatorname{cof}(|G|) \geq \kappa$, then we can construct $\mathcal{J}$ to be $\kappa$-complete.

Proof. It is easy to see that

$$
\mathcal{J}^{*}=\{-X: \forall A \in \mathcal{J} X+A \neq G\} .
$$

We keep this in mind through the rest of this proof. Let $\mathcal{F}$ be the family of subsets of $G$ produced in Theorem 1.1 for $\kappa$ and for $\mathcal{I}=\{\emptyset\}$. Let $\mathcal{J}$ be the translation invariant ideal generated by $\mathcal{F}$. The conclusion holds by Theorem 1.1 (ii) and (iii). Assume $\operatorname{cof}(|G|) \geq \kappa$. Let $\mathcal{J}$ be the translation invariant, $\kappa$-complete ideal generated by $\mathcal{F}$. Now the conclusion follows by Theorem 1.1 (i) and (ii).

Acknowledgement. I would like to thank the referee for suggestions which improved the presentation of the material contained in the paper. I also thank P. Zakrzewski for pointing out a mistake in an earlier version of the paper.

\footnotetext{
${ }^{1}$ Actually, Seredyński asked whether $\{$ countable subsets of $\mathbb{R}\}$ is $* *$-closed. A family $\mathcal{I}$ is $* *-$ closed if $\mathcal{I}^{* *}=\mathcal{I}$. However, by $\left[12\right.$, p. 206], $\mathcal{I}^{* * *}=\mathcal{I}^{*}$ for any $\mathcal{I}$. It follows now that $\mathcal{I}$ is **-closed if, and only if, there exists a family of sets $\mathcal{J}$ with $\mathcal{I}=\mathcal{J}^{*}$.
} 


\section{REFERENCES}

[1] T. Bartoszyński and H. Judah, Set Theory. On the Structure of the Real Line, A K Peters, 1995.

[2] T. Carlson, Strong measure zero and strongly meager sets, Proc. Amer. Math. Soc. 118 (1993), 577-586.

[3] J. Cichoń, A. Kharazishvili, and B. Wȩglorz, On sets of Vitali's type, Proc. Amer. Math. Soc. 118 (1993), 1243-1250.

[4] P. Erdős, K. Kunen, and R.D. Mauldin, Some additive properties of sets of real numbers, Fund. Math. 113 (1981), 187-199.

[5] P. Erdős and R.D. Mauldin, The nonexistence of certain invariant measures, Proc. Amer. Math. Soc. 59 (1976), 321-322.

[6] H. Friedman and M. Talagrand, Un ensemble singulier, Bull. Sci. Math. 104 (1980), 337340.

[7] F. Galvin, J. Mycielski, and R.M. Solovay, Strong measure zero sets, Notices Amer. Math. Soc. 26 (1973), A-280.

[8] A.B. Kharazishvili, On some types of invariant measures, Soviet Math. Dokl. 16 (1975), 681-684.

[9] A.B. Kharazishvili, Transformation Groups and Invariant Measures. Set Theoretical Aspects, World Scientific, 1998.

[10] R. Laver, On the consistency of Borel's conjecture, Acta. Math. 137 (1976), 151-169.

[11] A. Nowik, On a measure which measures at least one selector for every uncountable group, Real Anal. Exchange 22 (1996/97), 814-817.

[12] W. Seredyński, Some operations related to translations, Colloq. Math. 57 (1989), 203-219.

[13] S. Shelah, Every null additive set is meager additive, Israel J. Math. 89 (1995), 357-376.

[14] S. Solecki, Measurability properties of sets of Vitali's type, Proc. Amer. Math. Soc. 119 (1993), 897-902.

[15] E. Szpilrajn (Marczewski), Sur l'extension de la mesure lebesguienne, Fund. Math. 25 (1935), 551-558.

Department of Mathematics, 1409 W. Green Street, University of Illinois, UrBANA, IL 61801

E-mail address: ssolecki@math.uiuc.edu 